\title{
Hydrogen-bonding Interactions of $C$-Tetraundecenylresorc[4]arene with Some Guest Molecules
}

\author{
Mizuho Maeda ${ }^{1,2}$, Tadashi Aoyama ${ }^{1}$, Toshio TAKIdo ${ }^{*}$ and Manabu Seno ${ }^{1}$ \\ ${ }^{1}$ College of Science and Technology, Nihon University \\ (Kanda Surugadai 1-8-14, Chiyoda-ku, Tokyo 101-8308, JAPAN) \\ ${ }^{2}$ Department of Environmental Chemistry, Tokyo Institute, Polytechnic University \\ (Ogawanishimachi 2-32-1, Kodaira, Tokyo 187-0035, JAPAN)
}

Accepted July 31, 2006 (received for review May 12, 2006)

\begin{abstract}
H}-\mathrm{NMR}$ measurements show that the modes of hydrogen-bonding interaction of $C$-tetraundecenylresorc[4]arene with the guest molecules are classified into four groups, depending on whether the guest molecule is a hydrogen-bond donor, a hydrogen-bond acceptor or works as both donor and acceptor.
\end{abstract}

Key words: hydrogen bond, hydrogen-bonding interaction, acceptor, donor, resorc[4]arene

\section{Introduction}

Resorc[4]arene is a cyclic tetramer prepared by the cyclo-condensation reaction of resorcinol with various aldehydes catalyzed by acid. Resorc[4]arene has a hydrophobic molecular cavity surrounded by four benzene rings, which includes various organic guest molecules such as toluene (1-3). Along the upper rim of the cavity, eight hydroxyl groups are arranged to form a polar part of the molecule. Resorc[4]arene extracts some polar molecules such as sugars from a nonpolar medium through interactions with these hydroxyl groups, as studied extensively by Y. Aoyama et al. (4). Moreover, various derivatives were prepared by the chemical modification of the hydroxyl groups; for example, the cavitand was prepared by the crosslinking of adjacent hydroxyl groups by quinoxaline or bromochloromethane (5-9).

The hydroxyl groups along the upper rim form an intramolecular network of hydrogen bonds, stabilizing the cone conformation of the molecule. These hydroxyl

\section{Abbreviations:}

acceptor number (AN), $C$-tetraundecenylresorc[4]arene (TUresorc[4]arene), donor number (DN) groups are also involved in the formation of molecular assemblies by making intermolecular hydrogen bonds in nonpolar solvents as shown in Fig. 1 (10-12). As shown by these examples, the state of the hydroxyl groups has great impact on the properties of resorc[4]arene. In this paper, we will report the results of ${ }^{1} \mathrm{H}$-NMR measurements on $C$-tetraundecenylresorc[4]arene (TUresorc[4]arene) in various deuterated solvents and in the presence of some guest molecules in order to clarify the state of hydroxyl groups under various conditions, which is an important issue underlining the molecular recognition phenomena of resorc[4]arene.

\section{Experimental}

\section{$\mathbf{2} \cdot \mathbf{1}$ Materials}

TUresorc[4] arene was prepared according to the method reported in the literature (9). Deuterated-benzene $\left(\mathrm{C}_{6} \mathrm{D}_{6}\right)$ in ampules for ${ }^{1} \mathrm{H}-\mathrm{NMR}$ measurements was obtained from Acros Organics Company, and other deuterated solvents $\left(\mathrm{CDCl}_{3}\right.$, acetone- $d_{6}$, acetonitrile- $d_{3}$, and dimethylsulfoxide- $d_{6}$ (DMSO- $d_{6}$ ) were obtained from Merck Company. Acetone, acetonitrile, butyralde-

\footnotetext{
* Correspondence to: Toshio TAKIDO, College of Science and Technology, Nihon University, Kanda Surugadai 1-8-14, Chiyoda-ku, Tokyo 1018308, JAPAN

E-mail: takido@chem.cst.nihon-u.ac.jp
} 


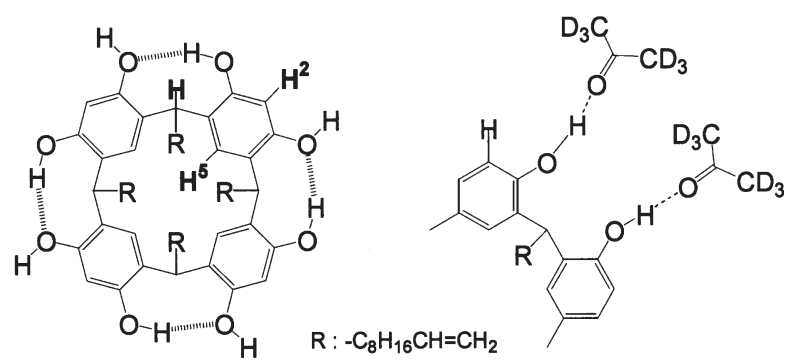

(a) in $\mathrm{C}_{6} \mathrm{D}_{6}$

(b) in acetone- $d_{6}$
Fig. 1 Intramolecular Hydrogen-bonding Network

(a) and Intermolecular Hydrogen Bonds

(b) of TUresorc[4]arene.

hyde, methanol, 1-propanol, 2-propanol, 1-hexanol, $m$ cresol, 1-hexylamine, triethylamine, aniline, pyridine, DMSO, N,N-dimethylformamide (DMF), chloroform, and water, which were used as guest substances, were obtained from Kanto Kagaku and Wako Pure Chemical Industries, and were used without further purification.

\section{$2 \cdot 2$ Measurements}

${ }^{1} \mathrm{H}-\mathrm{NMR}$ spectra were recorded on a Jeol JNMGX400 (400MHz) by using TMS as an internal standard. The measurements were carried out at ambient temperature $\left(20 \pm 1^{\circ} \mathrm{C}\right)$ unless otherwise stated. The IR spectra were recorded with a Shimadzu FTIR-8100 by the KBr method.

\subsection{Typical Procedure}

$2 \cdot 3 \cdot 1$ Hydrogen-bonding states of TUresorc[4]arene in various deuterated solvents

TUresorc[4]arene $(10.4 \mathrm{mg}, 10 \mu \mathrm{mol})$ was dissolved in $\mathrm{C}_{6} \mathrm{D}_{6}$ or $\mathrm{CDCl}_{3}(0.6 \mathrm{~mL})$ and the Chemical shift of hydroxyl protons of TUresorc[4]arene was measured by using ${ }^{1} \mathrm{H}-\mathrm{NMR}$.

$2 \cdot 3 \cdot 2$ Hydrogen-bonding interaction of TUresorc[4]arene with some guest molecules ( ${ }^{1}$ H-NMR measurements)

Given amounts of each guest were added into a solution of TUresorc[4]arene (10.4 mg) in $0.6 \mathrm{ml}$ of $\mathrm{C}_{6} \mathrm{D}_{6}$ to assure the molar ratio between 1:0 and 1:100, and ${ }^{1} \mathrm{H}$ NMR signals were compared.

$2 \cdot 3 \cdot 3$ Hydrogen-bonding interaction of TUresorc[4]arene with some guest molecules (IR measurements)

Given amounts of methanol or acetone were added to TUresorc[4]arene in a $\mathrm{KBr}$ disk to adjust the molar ratio to be $1: 0,1: 4,1: 32$, and 1:64 and IR spectra in the range in $3000-3500 \mathrm{~cm}^{-1}$ were compared.

\section{Results and Discussion}

\section{$3 \cdot 1$ Hydrogen-Bonding States of TUresorc[4]arene in Various Deuterated Solvents}

The signals of hydroxyl protons in the ${ }^{1} \mathrm{H}-\mathrm{NMR}$ spectra of TUresorc[4]arene were investigated in various deuterated solvents, the results of which are summarized in Table 1. Two signals of hydroxyl protons are observed in the ${ }^{1} \mathrm{H}-\mathrm{NMR}$ spectra of TUresorc[4] arene in $\mathrm{C}_{6} \mathrm{D}_{6}$ and $\mathrm{CDCl}_{3}(13,14)$. The lower-field signal is assigned to the hydroxyl protons forming the intramolecular hydrogen-bonding network and the other upper-field signal is assigned to "free" hydroxyl protons, which are in a nearly free state or in a intermolecular hydrogen-bonded state forming weak molecular assemblies (12). These signals are shifted slightly upfield in $\mathrm{CDCl}_{3}$ compared with those in $\mathrm{C}_{6} \mathrm{D}_{6}$; this may be owing to weakened intermolecular hydrogen bonds by a polar effect of $\mathrm{CDCl}_{3}$.

Resorcinol exhibits only a peak assigned to hydroxyl groups at $8.15 \mathrm{ppm}$ (acetone- $d_{6}$ ) and $9.15 \mathrm{ppm}$ (DMSO$d_{6}$ ), respectively, which confirms the equivalence of two hydroxyl groups. The formation of intramolecular hydrogen-bonding network is peculiar to resorc[4]arene owing to its characteristic steric structure.

In acetone- $d_{6}$, acetonitrile- $d_{3}$, and DMSO- $d_{6}$, the two signals collapse into a single signal, which shows that the hydrogen bonding network becomes labile and, therefore, eight hydroxyl protons can not be discriminated from each other. TUresorc[4]arene interacts with these polar solvent molecules (Fig. 1b). The mode of interactions of the hydroxyl groups with these molecules are detailed in later sections.

The relationship between the chemical shift of the hydroxyl protons and the relative dielectric constant of solvent is shown in Fig. 2. A good correlation is observed except for DMSO- $d_{6}$. This shows that the intramolecular hydrogen bonds are weakened with increasing relative dielectric constant of the solvent. In DMSO- $d_{6}$, the chemical shift deviates from this trend, appearing rather downfield $(8.85 \mathrm{ppm})$ in spite of its high relative dielectric constant (46.7). DMSO- $d_{6}$ molecules are hydrogen-bonded to the hydroxyl protons of TUresorc[4]arene via the sulfoxide groups, as 
Table $1{ }^{1}$ H-NMR Signals Assigned to the Hydroxyl Protons of TUresorc[4]arene in Some Deuterated Solvents.

\begin{tabular}{|c|c|c|c|c|c|c|c|c|}
\hline \multirow{3}{*}{$\begin{array}{l}\text { Deuterated Solvent } \\
\mathrm{C}_{6} \mathrm{D}_{6}\end{array}$} & \multicolumn{7}{|c|}{ Cemical Shift (ppm) } & \multirow{3}{*}{ 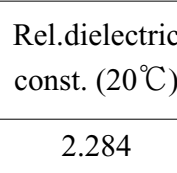 } \\
\hline & \multicolumn{2}{|c|}{$\mathrm{Ar}-\mathrm{OH}$} & \multirow{2}{*}{$\begin{array}{c}\text { Ar-CHR-Ar } \\
4.72 \text { (br.s) }\end{array}$} & \multirow{2}{*}{$\begin{array}{r}\text { Ar- } H^{2} \\
6.39\end{array}$} & \multirow{2}{*}{$\begin{array}{c}\text { Ar- } H^{4} \\
-\end{array}$} & \multirow{2}{*}{$\begin{array}{c}\mathrm{Ar}-H^{5} \\
7.73\end{array}$} & \multirow{2}{*}{$\begin{array}{c}\mathrm{Ar}-H^{6} \\
-\end{array}$} & \\
\hline & 9.94(br.s) & 9.51 (br.s) & & & & & & \\
\hline $\mathrm{CDCI}_{3}$ & 9.61 (br.s) & 9.28 (br.s) & $4.29(\mathrm{t}, J=7.6 \mathrm{~Hz})$ & 6.11 & - & 7.19 & - & 4.806 \\
\hline Acetone- $d_{6}$ & \multicolumn{2}{|c|}{8.46} & $4.31(\mathrm{t}, J=7.6 \mathrm{~Hz})$ & 6.22 & - & 7.55 & - & $20.7 * 1)$ \\
\hline Acetone- $\left.d_{6} * 2\right)$ & \multicolumn{2}{|c|}{8.15} & - & 6.35 & $6.33(d)$ & $6.98(t)$ & $6.33(d)$ & $20.7 * 1)$ \\
\hline Acetonitrile- $d_{3}$ & \multicolumn{2}{|c|}{7.58} & $4.16(\mathrm{t}, J=7.6 \mathrm{~Hz})$ & 6.21 & - & 7.25 & - & 37.5 \\
\hline DMSO- $d_{6}$ & \multicolumn{2}{|c|}{8.85} & $4.21(\mathrm{t}, J=7.6 \mathrm{~Hz})$ & 6.14 & - & 7.11 & - & 46.7 \\
\hline $\left.\mathrm{DMSO}-d_{6} * 2\right)$ & \multicolumn{2}{|c|}{9.15} & - & 6.19 & $6.18(d)$ & $6.91(\mathrm{t})$ & $6.18(\mathrm{~d})$ & 46.7 \\
\hline
\end{tabular}

*1) $25^{\circ} \mathrm{C}$

*2) resorcinol

described later.

The effect of temperature on the chemical shift of hydroxyl protons of TUresorc[4] arene in $\mathrm{C}_{6} \mathrm{D}_{6}$ is shown in Fig. 3a. The chemical shift moves slightly upfield, showing weakened hydrogen bonds, with increasing temperature. Two signals observed at temperatures lower than $70^{\circ} \mathrm{C}$ coalesced into a single broad signal at $80^{\circ} \mathrm{C}$.

The rate constant and the activation free energy for the proton exchange reaction between the protons in the intramolecular hydrogen-bonding network and the "free" protons were estimated by a total band-shape
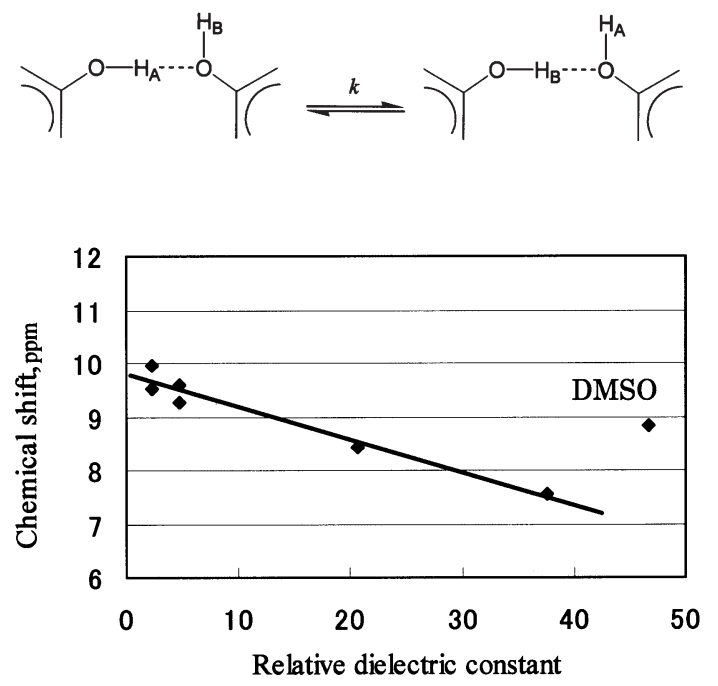

Fig. 2 Relationship of the Chemical Shift of the Hydroxyl Protons and the Relative Dielectric Constant of Solvent. analysis (15) using the hydroxyl signals. In the calculation, the spectrum is approximated by two signals separated by $156 \mathrm{~Hz}$. It was assumed that the transverse relaxation time $\left(T_{2}\right)$ is so large that the broadening of the line shape is caused exclusively by the exchange reaction. The results of the simulation of the line-shape obtained by fitting to the observed spectra are included in Fig. 3, together with the determined values of $k$. Despite some variation in the value of $k$, there is a clear trend that the exchange rate increases at higher temperatures. These $k$ values, according to the activation complex theory, give an activation free energy of 60-70 $\mathrm{kJmol}^{-1}$ for the proton exchange reaction.

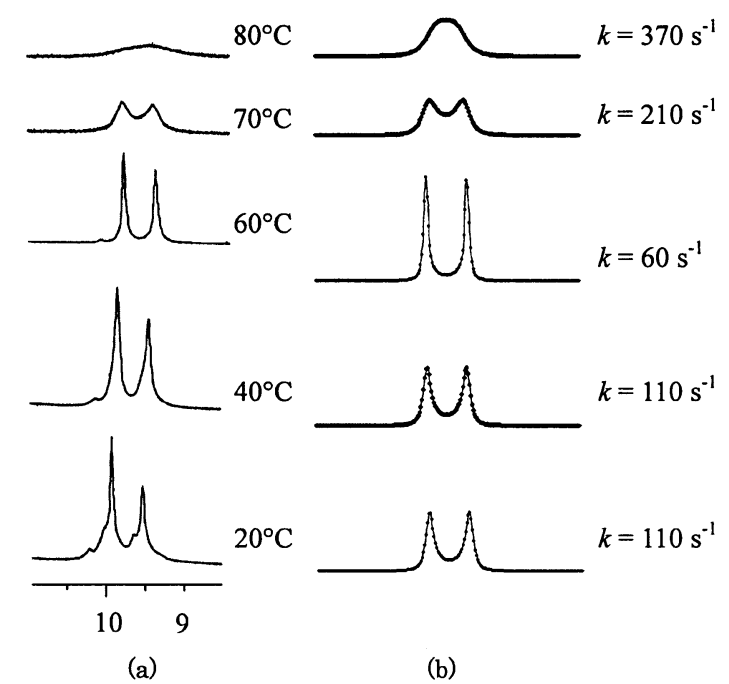

Fig. 3 Temperature Dependence of the Hydroxyl Proton Signals. a : TUresorc[4] arene in $\mathrm{C}_{6} \mathrm{D}_{6}, \mathrm{~b}$ : the result of simulation. 


\section{$3 \cdot 2$ Hydrogen-Bonding Interaction of TUresorc[4]arene with Some Guest Molecules}

$3 \cdot 2 \cdot 1 \quad{ }^{1} \mathrm{H}-\mathrm{NMR}$ study on hydroxyl protons

The ${ }^{1} \mathrm{H}-\mathrm{NMR}$ spectra were examined for the hydrox$\mathrm{yl}$ protons of TUresorc[4]arene in the presence of varied amounts of guest molecules in $\mathrm{C}_{6} \mathrm{D}_{6}$. Some substances which are expected to interact with hydroxyl groups of TUresorc[4] arene and are soluble in $\mathrm{C}_{6} \mathrm{D}_{6}$ were selected. The molar ratio 1:8 corresponds to the equimolar mixture for the host and guest, as TUresorc[4]arene has eight hydroxyl groups per molecule. The ${ }^{1} \mathrm{H}-\mathrm{NMR}$ data are summarized in Table 2, in which the chemical shift values of all the protons of TUresorc[4]arene in $\mathrm{C}_{6} \mathrm{D}_{6}$ with added guest compounds of the molar ratio 1:8 are shown. The chemical shift values change with the added amount of guest in the mode characteristic of the kind of guest. The typical data are displayed in Fig.4.

Group 1: hydrogen-bond acceptor molecules (A)

The ${ }^{1} \mathrm{H}$ NMR spectra in this group has the common feature that a singlet signal appears upon the addition of guest molecules. This new signal becomes sharper in the presence of excess guest molecules. This group contains hydrogen-bond acceptors.

\section{(a) Acetone}

When incremental amounts of acetone were successively added to a TUresorc[4] arene solution in $\mathrm{C}_{6} \mathrm{D}_{6}$ at room temperature, the ${ }^{1} \mathrm{H}-\mathrm{NMR}$ spectra changed as shown in Fig. 4a. By the addition of acetone, the initial two signals were slightly shifted downfield; the upperfield signal was shifted more. A new signal appeared at about 8 ppm when more than 8 equivalents (with regard to the hydroxyl group) of acetone were added, and grew up with increasing amount of acetone. When a large excess of acetone was added, the original signals at about $10 \mathrm{ppm}$ disappeared and only the new signal at about 8 ppm was observed, as was observed for TUresorc[4] arene in acetone- $d_{6}$ (Table 1). This signal may be assigned to a state in which all hydroxyl groups of TUresorc[4] arene are hydrogen-bonded to acetone molecules, as shown in Scheme 1 (and also in Fig. 1b). The change corresponds to the transition from 'state a'to 'state c' in Scheme 1. The ${ }^{1} \mathrm{H}-\mathrm{NMR}$ charts in Fig. 4a represent a gradual equilibrium shift from 'state a' to 'state c'. The transient 'state b' was not clearly observed by ${ }^{1} \mathrm{H}-\mathrm{NMR}$.

(b) Acetonitrile
The spectral change upon the addition of acetonitrile is shown in Fig. 4b, which is very similar to the case of acetone. This shows a similarity in the interaction modes with these molecules. However, the transition from 'state a' to 'state c' in Scheme $\mathbf{1}$ is less favored as a new signal appears upon the addition of more than 50 equivalents. This may be owing to a weaker ability as a hydrogen-bond acceptor of acetonitrile. It is consistent with the fact that the donor number (DN) of acetonitrile (14.1) is smaller than that of acetone (17.0). The DN value proposed by Gutman (16) represents the ability as a donor of an unshared electron pair and, therefore, the

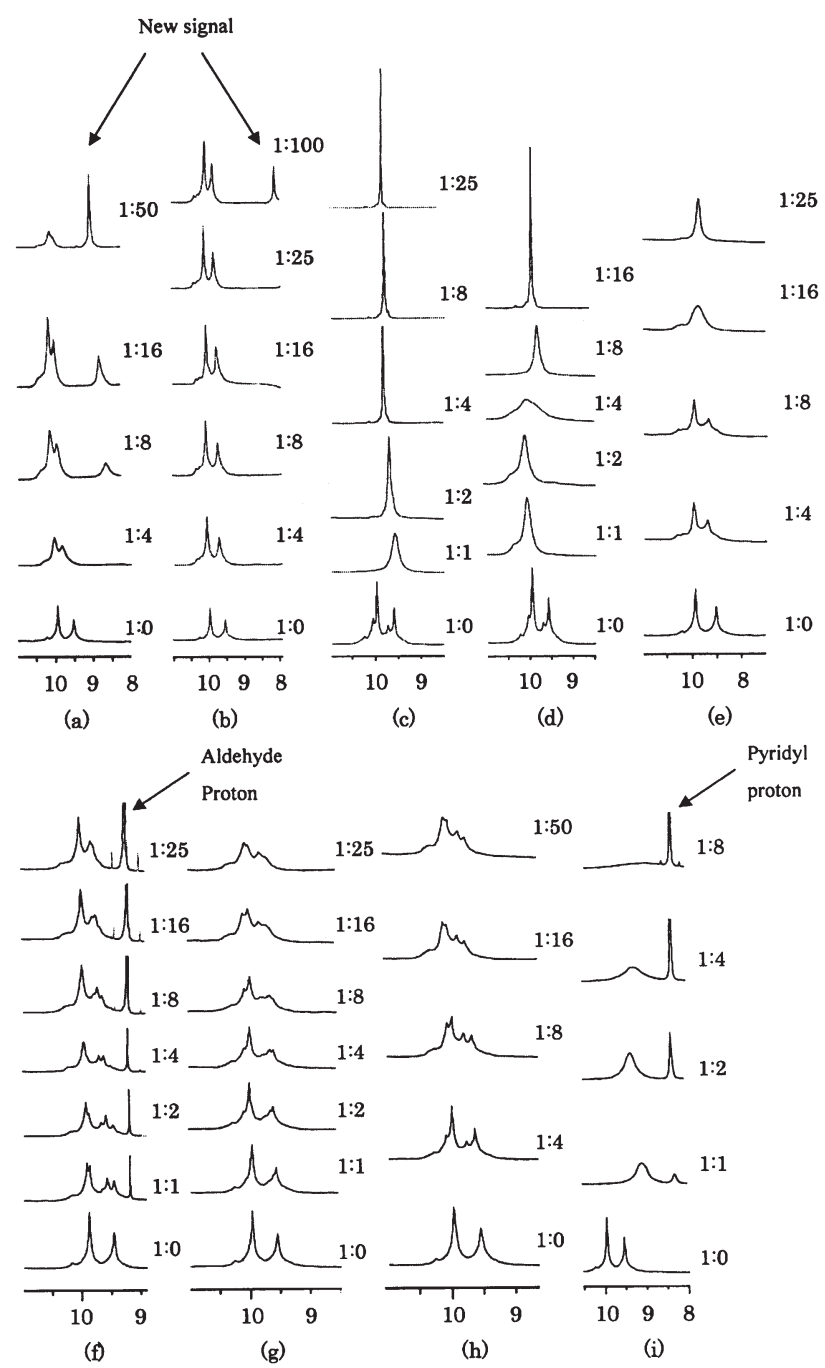

Fig. $4{ }^{1}$ H-NMR Spectra in the Range of Hydroxyl Protons in the Presence of Some Guest Molecules. a: acetone, b: acetonitrile, c: DMSO, d: DMF, e: methanol, f: butyraldehyde, g: $m$-cresol, h: water (the ratios are based on the feed amounts, since $\mathrm{H}_{2} \mathrm{O}$ and $\mathrm{C}_{6} \mathrm{D}_{6}$ are not completely miscible), i: pyridine. 
Table $2{ }^{1}$ H-NMR Signals of TUresorc[4] arene with Added Various Guest Compounds at the Molar Ratio of 1:8 in $\mathrm{C}_{6} \mathrm{D}_{6}$.

\begin{tabular}{|c|c|c|c|c|c|c|}
\hline Guests & \multicolumn{3}{|c|}{$\mathrm{Ar}-\mathrm{OH}$} & $\mathrm{Ar}-H^{5}$ & $\mathrm{Ar}-H^{2}$ & Ar-CHR-Ar \\
\hline (a) Acetone & 9.90 (br.s) & 9.80 (br.s) & 8.40 (br.s) & 7.65 & 6.52 & 4.68(br.s) \\
\hline (b) Acetonitrile & 10.0 (br.s) & 9.70(br.s) & - & 7.68 & 6.53 & 4.70(br.s) \\
\hline (c) DMSO & \multicolumn{2}{|c|}{9.82} & - & 7.63 & 6.95 & $4.81(\mathrm{t}, J=7.6 \mathrm{~Hz})$ \\
\hline (d) DMF & \multicolumn{2}{|c|}{ 9.81(br.s) } & - & 7.66 & 6.92 & $4.81(\mathrm{t}, J=7.6 \mathrm{~Hz})$ \\
\hline (e) Methanol & 10.0 (br.s) & 9.74(br.s) & - & 7.70 & 6.24 & 4.71(br.s) \\
\hline (f) Butyraldehyde & 10.0 (br.s) & 9.77(br.s) & - & 7.68 & 6.47 & 4.71(br.s) \\
\hline (g) $m$-Cresole & 9.97(br.s) & 9.63(br.s) & - & 7.70 & $* 1)$ & 4.71(br.s) \\
\hline (h) Water & 9.96(br.s) & 9.78 (br.s) & - & 7.70 & 6.41 & 4.70(br.s) \\
\hline (i) Pyridine & \multicolumn{2}{|c|}{$9.10(\mathrm{brs}) * 2)$} & - & 7.74 & 6.43 & $4.88(\mathrm{t}, J=7.6 \mathrm{~Hz})$ \\
\hline
\end{tabular}

the molar ratio of a guest against TUresorc[4]arene is 1:8

*1) not detemined

*2) the molar ratio 1: 4

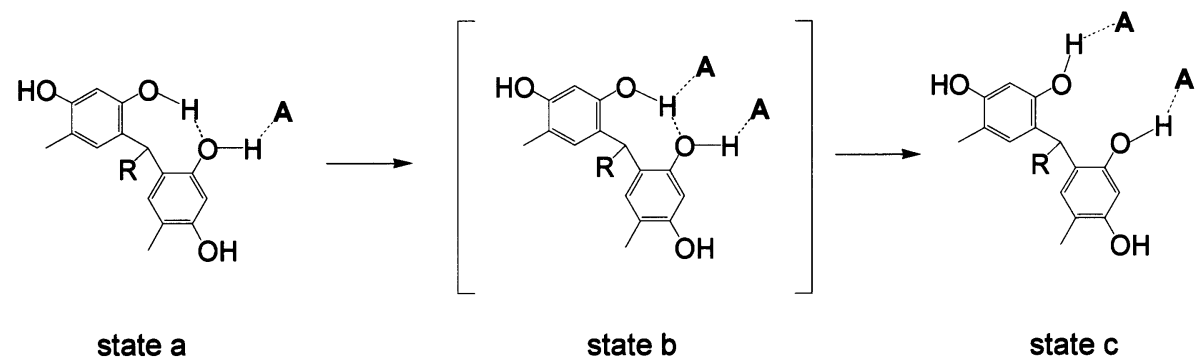

Scheme 1 Hydrogen-Bonding Interactions of the Hydroxyl Groups of TUresorc[4] arene with Hydrogen-Bond Acceptor Molecules A.

higher the DN value, the higher the ability for accepting the hydroxyl protons.

\section{(c) DMSO}

The addition of DMSO gave an interesting result, as shown in Fig. 4c. By the addition of one equivalent of DMSO, the original lower-field signal disappeared leaving behind the broadened upper-field signal, which became sharper with a slight downfield shift by further addition. This change may be essentially explained by Scheme 1, which was proposed for the case of acetone. Compared with the case of acetone, the transition from 'state a' to 'state c' is much more rapid (and the transit 'state b' was not detected), since DMSO is a stronger hydrogen-bond acceptor than acetone, as indicated by the $\mathrm{DN}$ value (29.6).

(d) DMF

The effect of the addition of DMF was similar to the case of DMSO, as shown in Fig. 4d. By the addition of one equivalent of DMF, the original two signals collapsed into one broad signal with a downfield shift. This signal disappeared at the molar ratio 1:8, and a new sharp signal appeared at a slightly upfield side. These changes may be represented by Scheme 1. This transition occurs at the molar ratio of 1:1 in the case of DMSO and this reflects a higher DN value of DMSO compared to that of DMF (26.6).

Group 2: hydrogen-bond donor molecules (BH)

Aliphatic alcohols constitute Group 2. Only the signal for the "free" hydroxyl protons shifts downfield upon the addition of alcohols.

(e) Alcohols

Alcohols used were methanol, 1-propanol, 2propanol, and 1-hexanol. Spectral changes by the addition of methanol are shown in Fig. 4e. Clearly, the pattern of change in the case of methanol is different from those in Group 1; that is, there appeared no new signal. 
There was no splitting of the signals either, which was observed for Group 3 as described below. By the addition of 16 equivalents of methanol, the two signals collapsed into a broad signal without a large change in the chemical shift. This mode of change may be interpreted by the change in hydrogen-bonding structure as shown in Scheme 2. The effects of the addition of other alcohols are summarized in Fig. 5. The mode of change is essentially the same as in the case of methanol, but the signals shift slightly downfield at first, and then move upfield gradually by the addition of these alcohols. The initial downfield shifts show that the hydroxyl protons of TUresorc[4]arene are involved in stronger hydrogen bonding, while the upfield shift afterward corresponds to an equilibration between bulk alcohol OHs. There is a trend toward splitting again after collapsing into a single signal in the presence of excess 2-propanol and 1hexanol.

The hydroxyl group in alcohols can, in principle, work not only as a hydrogen-bond donor but also as a hydrogen-bond acceptor. However, the pattern of spectral changes upon the addition of alcohols is distinctly different from those in Group 3 described below, which is constituted with molecules which work as both a hydrogen-bond donor and an acceptor.

Group 3: guest molecules which work as both a hydrogen-bond donor and an acceptor (AH)
As for the guests of Group 3, the original two signals of hydroxyl protons have the tendency of spliting into doublets or multiplets.

(f) Butyraldehyde

The result of the addition of butyraldehyde is shown in Fig. 4f. Each of the original two signals splitted into a doublet and a new signal did not appear, being different from Group 1 and 2. This may be owing to the presence of the aldehyde proton besides the carbonyl group. The aldehyde group serves as a hydrogen-bond donor as well as a hydrogen-bond acceptor, as shown in Scheme 3. It was reported by C. F. Wilson et al. (13) that the hydroxyl protons of resorc[4]arene appear in the ${ }^{1} \mathrm{H}-\mathrm{NMR}$ spectrum as a pair of doublets at temperatures below $45^{\circ} \mathrm{C}$, due to an asymmetry in the hydrophilic rim with an associated conformational change. Hence, the splitting in this case may also be due to an asymmetry caused by hydrogen bonds formed between TUresorc[4] arene and butyraldehyde.

(g) $m$-Cresol

The original two signals became gradually broad and splitted into doublets upon the addition of $m$-cresol as shown in Fig. 4g. These signals remained until the addition up to the molar ratio $1: 100$, suggesting the presence of the intramolecular hydrogen-bonding network under these conditions. This is because the coordination of acidic proton of $m$-cresol to the hydroxyl

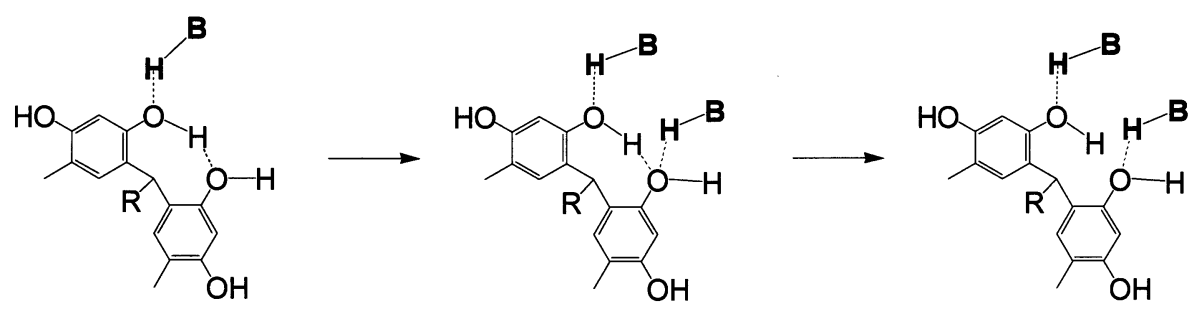

Scheme 2 Hydrogen-Bonding Interactions of the Hydroxyl Groups of TUresorc[4]arene with Hydrogen-Bond Donor Molecules BH.

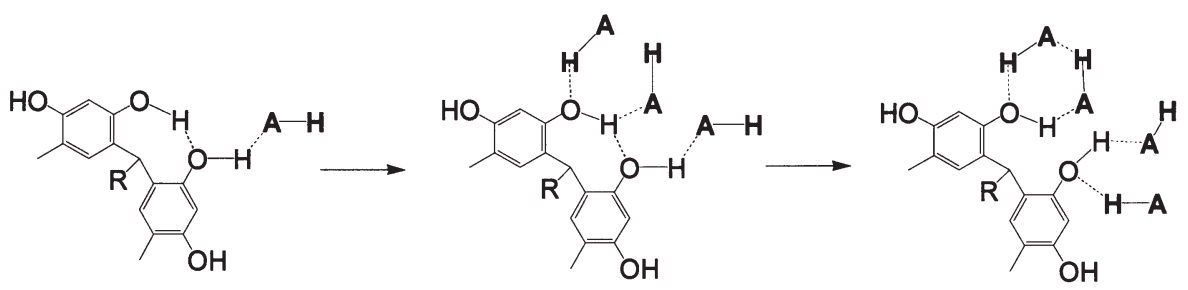

Scheme 3 Hydrogen-Bonding Interactions of the Hydroxyl Groups of TUresorc[4]arene with Hydrogen-Bond Acceptor and Donor Molecules AH. 

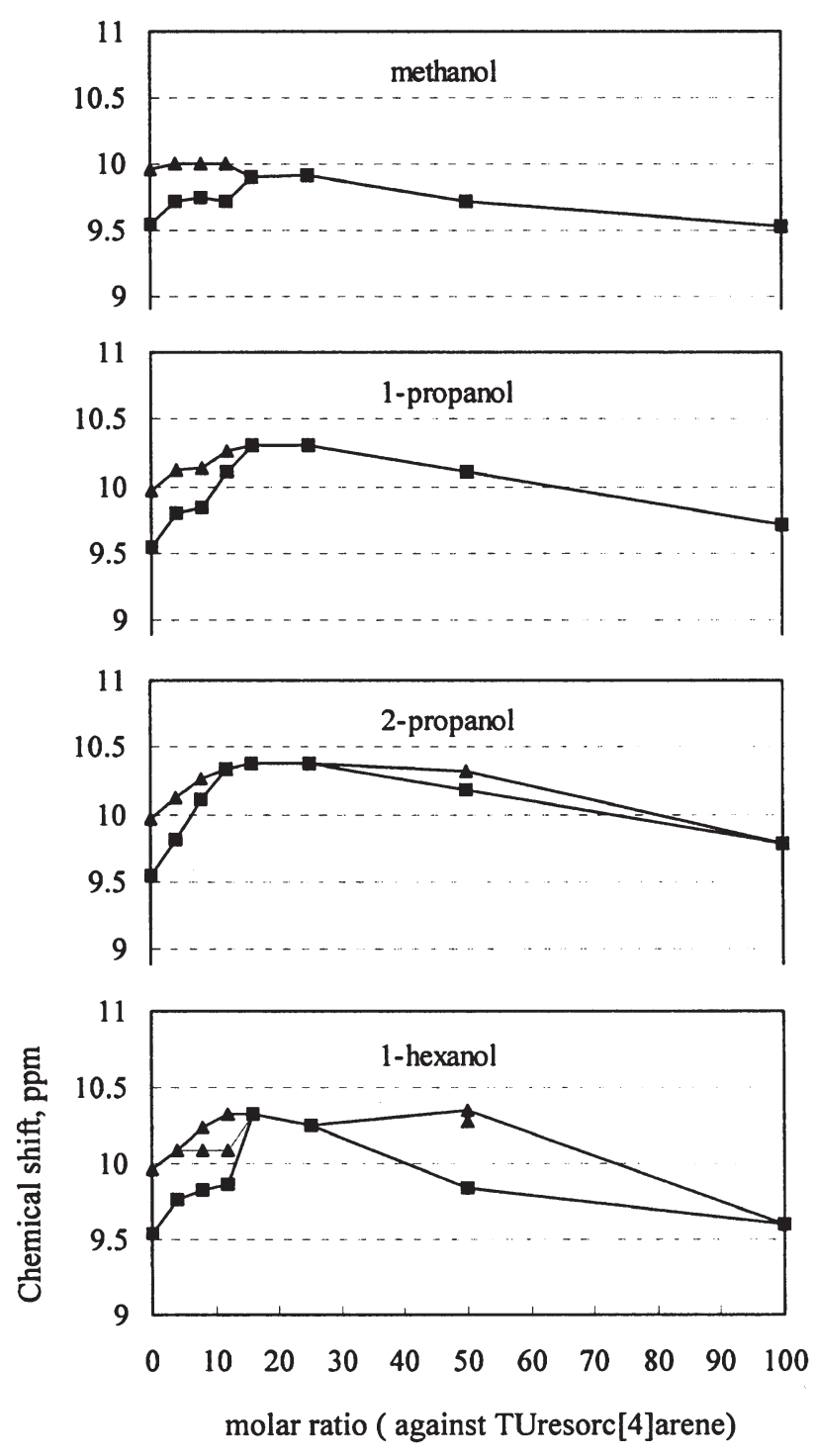

Fig. $5{ }^{1} \mathrm{H}$-NMR Spectral Changes by the Addition of Various Alcohols.

oxygen does not affect the intramolecular hydrogen bonding as depicted in Scheme 3 .

(h) Water

Water is immiscible with $\mathrm{C}_{6} \mathrm{D}_{6}$ but some quantities of water are immobilized on TUresorc[4]arene. The ${ }^{1} \mathrm{H}$ NMR spectra of TUresorc[4] arene with added water are shown in Fig. 4h. Each of the original two hydroxyl signals tends to split to a doublet (13), which is similar to the case of butyraldehyde and $m$-cresol as described above.

\section{Group 4: basic molecules $\left(\mathrm{BH}_{2}\right)$}

The signals for the guests, i.e., amines, in Group 4 became so broad up to the point where they are no longer observed in the ${ }^{1} \mathrm{H}-\mathrm{NMR}$ spectrum.

(i) Amines

$\mathrm{CDCl}_{3}$ was used as a solvent for the study of amines, because the sample solution became turbid when amines, such as hexylamine, were added into a $\mathrm{C}_{6} \mathrm{D}_{6}$ solution of TUresorc[4]arene. The addition of hexylamine, triethylamine, and aniline to a $\mathrm{CDCl}_{3}$ solution did not give turbidity, but the addition of diethylamine caused turbidity, being unable to measure ${ }^{1} \mathrm{H}-\mathrm{NMR}$ spectrum.

When hexylamine or triethylamine was added over a molar ratio of 1:4, the signals for the hydroxyl protons became so broad that they were no longer observed. In the case of aniline, the addition at the molar ratio 1:1 gave only a trace of broad signal. This could be interpreted as an acid-base reaction occurring between the acidic hydroxyl groups of TUresorc[4]arene and the basic amino group of amine, as being represented in Scheme 4. The Coulomb attraction between resulting phenoxide anions and ammonium cations leads to the formation of polar aggregates hardly soluble in nonpolar solvent. The proton exchange among these groups blur the ${ }^{1} \mathrm{H}-\mathrm{NMR}$ signals.

The spectral change by the addition of pyridine is shown in Fig. 4i. The addition of pyridine, a tertiary aromatic amine, gave a somewhat different result from the other amines. By the addition of pyridine at a molar ratio of $1: 1$, the original two signals combined into a broad signal with an upfield shift, which became broader by further addition until being smeared out with over

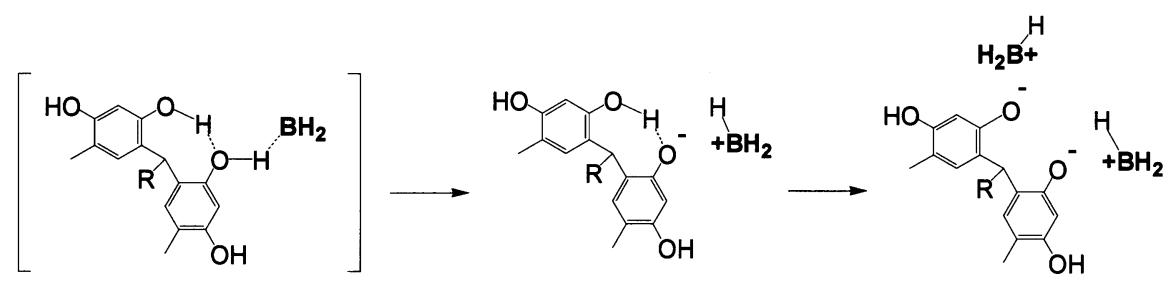

Scheme 4 Acid-Base Interactions of the Hydroxyl Groups of TUresorc[4] arene with Basic Molecules $\mathrm{BH}_{2}$. 
8 equivalents of pyridine.

The hydrogen-bonding interactions of TUresorc[4]arene with guest compounds shown in the preceding sections are thought to lead not a well-defined hydrogen-bonding complex of definite structure, but hydrogen-bonding clusters of loose structure. The extent of these interactions could be estimated only qualitatively from the peak change with the amount of added guest shown in Fig. 4.

\section{$3 \cdot 2 \cdot 2{ }^{1} \mathrm{H}-\mathrm{NMR}$ study on aromatic and methine protons of TUresorc[4]arene}

There was a common trend in the guest-induced chemical shift changes in the aromatic region: the addition of guest molecules in general tends to shift aromatic ortho protons (with respect to the hydroxyl group) downfield by a few ppm and meta protons only slightly upfield. In principle, chemical shift changes are caused either by a change in the electron density or in the conformation around the proton. The electronic effect caused by the hydrogen bond formation with guest molecules would increase the electron density in the aromatic rings, which should result in upfield shifts for aromatic protons. The downfield shifts actually observed for ortho protons thus suggest that the conformational change, rather than the electronic effect, has a larger effect on the chemical shift changes of the aromatic protons.

Some guest molecules caused the splitting of aromatic protons (DMF) or methine protons (propanol, hexanol, pyridine, and DMSO). The splitting indicates that these guest molecules, through hydrogen-bonding interactions, lock an asymmetric conformation of TUresorc[4]arene.

\section{$3 \cdot 2 \cdot 3$ IR spectra}

The IR spectra of the hydroxyl group region of TUresorc[4]arene with added methanol, acetone and hexylamine are shown in Fig. 6. The IR absorption by the hydroxyl groups of TUresorc[4]arene is observed as a broad band centered at $3280 \mathrm{~cm}^{-1}$. The hydroxyl group participated in the intramolecular hydrogen bonding network could not be discriminated from the free hydroxyl group. The addition of methanol does not affect the absorption band, but the addition of acetone makes the original band broader and shifted to the lower wave-number side. As described in a preceding section, methanol molecules are coordinated to the hydroxyl oxygen as a hydrogen-bond donor, and acetone molecules are coordinated to the hydroxyl proton as a hydrogen-bond acceptor. The former interactions would not disturb the hydrogen-bonding network as shown in Scheme 1, while the latter interactions would disrupt the hydrogen-bonding network as shown in Scheme 2. These changes of IR spectra are thus consistent with the models proposed based on ${ }^{1} \mathrm{H}-\mathrm{NMR}$ observations.

The addition of hexylamine gave a more remarkable change to the IR spectra of TUresorc[4]arene. By the

(a)

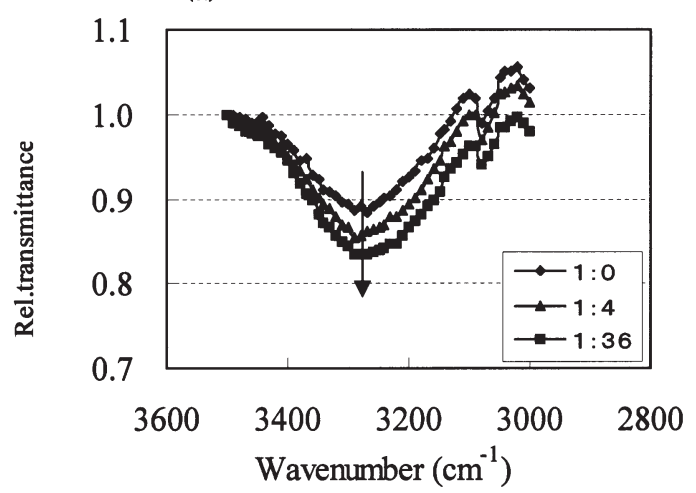

(b)

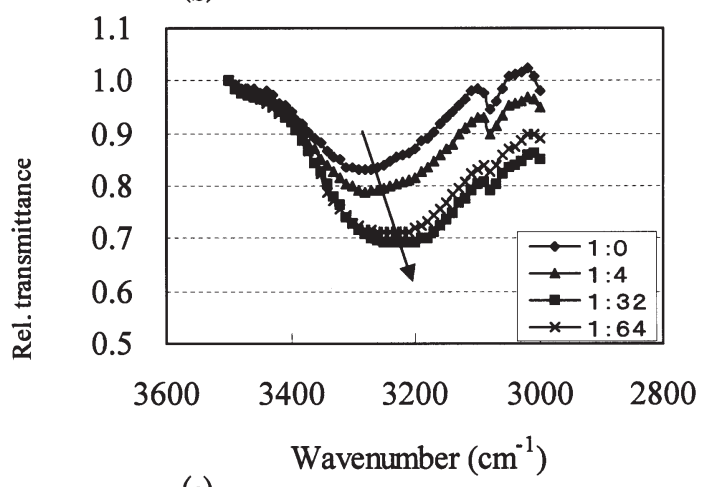

(c)

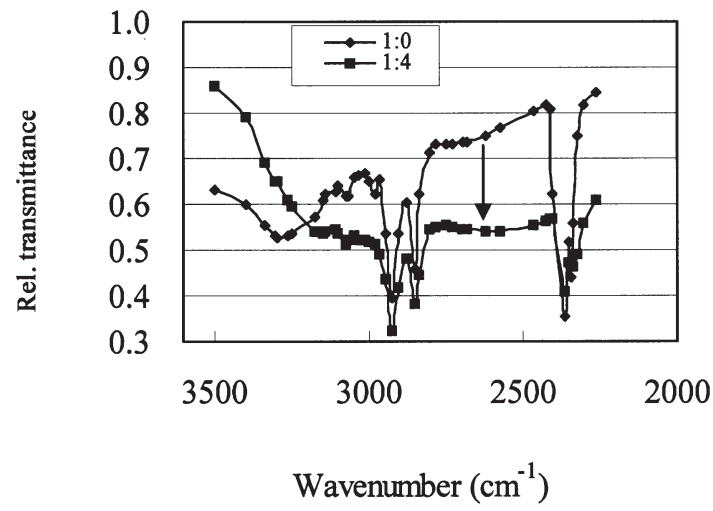

Fig. 6 IR Spectra in the Region of Hydroxyl Group of TUresorc[4]arene with Added Guest Substances. $\mathrm{a}:$ methanol, $\mathrm{b}$ : acetone, $\mathrm{c}:$ hexylamine. 
addition of four equivalents of hexylamine, the original band of hydroxyl groups was weakened and was shifted to around $3160 \mathrm{~cm}^{-1}$, and a broad band appeared between 2400 and $2800 \mathrm{~cm}^{-1}$; this band is assigned to the quaternary ammonium group produced by the proton shift from the hydroxyl group of TUresorc[4]arene to the amine. These spectral changes again agree with the picture shown in Scheme 4 based on the ${ }^{1} \mathrm{H}-\mathrm{NMR}$ study.

\section{Conclusion}

The guest molecules examined in this paper are broadly divided into three groups: Group 1: substances which act as a hydrogen-bond acceptor of the hydroxyl protons of TUresorc[4]arene; Group 2: substances which act as a hydrogen-bond donor to the hydroxyl oxygen; and Group 3: substances which play a roll of both a hydrogen-bond acceptor and a hydrogen-bond donor for the hydroxyl groups.

A typical substance in Group 1 is acetone; the mode of interaction with the hydroxyl groups is presented in Scheme 1. Acetonitrile, DMSO, and DMF are other examples. Their ability may be estimated in terms of the DN values. Amines have a very high ability as a hydrogen-bond acceptor. However, the proton exchange reaction becomes very rapid in the presence of amines, and thus the pattern of spectral changes are quite different from those in Group 1, as shown in Scheme 4.

Alcohols belong to Group 2; the hydrogen-bonding interaction with TUresorc[4]arene is as shown in Scheme 2. Their ability to form hydrogen bonds may be estimated in terms of the AN value; alcohols are stronger hydrogen-bond donor than chloroform, which hardly affects the ${ }^{1} \mathrm{H}$-NMR signals.

Butyraldehyde, $m$-cresol, and water show rather complicated behavior in the interaction with hydroxyl groups of TUresorc[4]arene. These substances have both a basic oxygen atom and an acidic hydrogen atom, thus work as both a hydrogen-bond acceptor and a hydrogen-bond donor, being categorized into Group 3. Their interactions with the hydroxyl groups of TUresorc[4]arene may be explained as shown in Scheme 3.

The classification of interactions of TUresorc[4]arene examined for the relatively simple molecules may also apply to more complex guest molecules which interact through hydrogen bond formation. Especially, analyzing the pattern of hydroxyl signals in ${ }^{1} \mathrm{H}-\mathrm{NMR}$ according to the criteria presented herein would help understand how the interactions occur in more elaborate host-guest systems.

\section{Acknowledgement}

This work is supported by the High-Tech Research Center of Nihon University. Authors thank Y. Okabe, $\mathrm{K}$. Yoda, and K. Ishiguro of Nihon University for their experimental assistance.

\section{References}

1. E. DALCANALE, G. COSTANTINI and P. SONCINI, Removal of Organic Pollutants from Water via Molecular Inclusion within a Cavitand, J. Incl. Phenom., Vol. 13, 87-92 (1992).

2. K.D. SCHIERBAUM, T. WEISS, E.U. THODEN VAN VELZEN, J.F.J. ENGBERSEN, D.N. REINHOUDT and W. GÖPEL, Molecular Recognition by Self-Assembled Monolayers of Cavitand Receptors, Science, Vol. 265, 1413-1415 (1994).

3. F.L. DICKERT, U.P.A. BÄUMLER and H. STATHOPULOS, Mass-Sensitive Solvent Vapor Detection with Calix[4]resorcinarenes: Tuning Sensitivity and Predicting Sensor Effects, Anal. Chem., Vol. 69, 1000-1005 (1997).

4. Y. TANAKA, Y. KATO and Y. AOYAMA, Two-Point HydrogenBonding Interaction: A Remarkable Chain-Length Selectivity in the Binding of Dicarboxylic Acids with Resorcinol- Aldehyde Cyclotetramer as a Multidentate Host, J. Am. Chem. Soc., Vol. 112, 2807-2808 (1990).

5. D.J. CRAM, S. KARBACH, H.E. KIM, C.B. KNOBLER, E.F. MAVERIC, J.L. ERICSON and R.C. HELGESON, Host-Guest Complexation. 46. Cavitands as Open Molecular Vessels Form Solvates, J. Am. Chem. Soc., Vol. 110, 2229-2237 (1988).

6. J.A. TUCKER, C.B. KNOBLER, K.N. TRUEBLOOD and D.J. CRAM, Host-Guest Complexation. 49. Cavitands Containing Two Binding Cavities, J. Am. Chem. Soc., Vol. 111, 3688-3699 (1989).

7. J.R. MORAN, J.L. ERICSON, E. DALCANALE, J.A. BRYANT, C.B. KNOBLER and D.J. CRAM, Vases and kites as Cavitands, J. Am. Chem. Soc., Vol. 113, 5707-5714 (1991).

8. D.J. CRAM, L.M. TUNSTAD and C.B. KNOBLER, C- and ZShaped Ditopic Cavitands, Their Binding Characteristics, and Monotopic Relatives, J. Org. Chem., Vol. 57, 528-535 (1992).

9. E.U. THODEN VAN VELZEN, J.F.J. ENGBERSEN and D.N. REINHOUDT, Synthesis of Self-Assembling Resorcin[4]arene Tetrasulfide Adsorbates, Synthesis, Vol. 8, 989-997 (1995).

10. Y. KIKUCHI, Y. KATO, Y. TANAKA, H. TOI and Y. AOYAMA, Molecukar Recognition and Stereoselectivity: Geometrical Requirements for the Multiple Hydrogen-Bonding Interaction of Diols with a Multidentate Polyhydroxy Macrocycle, J. Am. Chem. Soc., Vol. 113, 1349-1354 (1991).

11. K. KOBAYASHI, Y. ASAKAWA, Y. KIKUCHI, H. TOI and Y. 
AOYAMA, $\mathrm{CH}-\pi$ Interaction as an Important Driving Force of Host-Guest Complexation in Apolar Organic Media. Binding of Monools and Acetylated Compounds to Resorcinol Cyclic Tetramer As Studied by ${ }^{1} \mathrm{H}$ NMR and Circular Dichroism Spectroscopy, J. Am. Chem. Soc., Vol. 115, 2648-2654 (1993).

12. Y. AOYAMA, Y. TANAKA, H. TOI and H. OGOSHI, Polar Host-Guest Interaction. Binding of Nonionic Polar Compounds with a Resorcinol-Aldehyde Cyclooligomer as a Lipophilic Polar Host, J. Am. Chem. Soc., Vol. 110, 634-635 (1988).

13. C.F. WILSON, M.P. EASTMAN and C.J. HARTZELL, Hydrogen Bonding in a Host-Guest System: $C$-Undecylcalix[4]resorcinarene and Water in Benzene, J. Phys. Chem. B, Vol. 101,
9309-9313 (1997).

14. Y. AOYAMA, Y. TANAKA and S. SUGAHARA, Molecular Recognition. 5. Molecular Recognition of Sugars via HydrogenBonding Interaction with a Synthetic Polyhydroxy Macrocycle, J. Am. Chem. Soc., Vol. 111, 5397-5404 (1989).

15. H.S. GUTOWSKY and C.H. HOLM, Rate Processes and Nuclear Magnetic Resonance Spectra. II. Hindered Internal Rotation of Amides, J. Chem. Phys., Vol. 25, 1228-1234 (1956).

16. V. GUTMANN, Coordination Chemistry of Certain Transitionmetal Ions. Role of the Solvent, Coor. Chem. Rev, Vol. 2, 239256 (1967). 